\title{
Osteoarthritis is what the people have
}

\author{
Friedrich C. Luft ${ }^{1}$
}

Published online: 15 July 2015

(C) Springer-Verlag Berlin Heidelberg 2015

Degenerative joint disease, osteoarthrosis (in Europe), osteoarthritis (in the USA), and elsewhere, results from breakdown of joint cartilage and underlying bone. Joint pain and stiffness are the most common symptoms. The complaints are worsened by exercise and commonly become constant. Joint swelling, loss of joint motion range, and resultant weakness, numbness, and disability occur. The condition is asymmetrical. The joints involved are commonly distal, ends of fingers, base of the thumb, neck, lower back, knees, and hips. The condition afflicts work and daily activity. Unlike other forms of arthritis, the condition is confined to joints. Trauma, genetic predisposition, and overweight contribute to the disease. Inflammatory processes are intimately involved so that the term "arthritis" is very justified. The primary driving force is aging; all of us who survive will develop this problem, the sole question is when.

Trauma seems to be a predisposing factor for specific and premature osteoarthritis. For instance, the long-term consequences of anterior cruciate ligament and meniscus injuries and consequences of their treatment have been reviewed [1]. However, substantial "evidence-based" information gaps remain. There is little evidence to support a protective role of repair or reconstructive surgery against osteoarthritis development. In instances in which a randomized clinical trial is not feasible, natural history and other observational cohort studies could yield useful information. Interestingly, despite this dearth of clinical information, specific therapies have been

Friedrich C. Luft

luft@charite.de

1 Experimental and Clinical Research Center, Charité Medical Faculty and the Max Delbrück Center for Molecular Medicine,

Lindenbergerweg 80, 13125 Berlin, Germany spawned to avoid subsequent osteoarthritis after knee injuries. Autologous activated platelets retained in fibrin matrices are used as a source of molecular signals that control cell fate, including cell growth, cell differentiation, and the synthesis of diverse functional proteins. The idea has been developed that platelet-rich therapies (rich in growth-factor technologies) could be the source of advantageous signals [2]. The issues involve not only joint function but also (and perhaps more so) the consequences of joint osteoarthritis, namely pain syndromes. Osteoarthritis, low back pain, and fibromyalgia are common chronic pain disorders that occur frequently in the general population that are associated with aging [3]. Admittedly, genetic, environmental, and psychosocial factors likely play an important role. However, the economics of medical practices is surely also a contributor.

Aside from basketball and soccer players (USA-football players are currently more concerned about head-injury dementia), other elite performers present a challenge in terms of osteoarthritis. A delightful review by a physician who dedicated his career to help dancers and musicians has been published [4]. Artists in general are intelligent and the time spent on extensive explanation and advice is well spent, concluded the author. In overuse injuries, relative rest supported by "mental practice" is effective, the author advised. In terms of general physical exercise, there are few studies. An impressive case-control study with long-term follow up has been reported. About 500 aging (50-72 years) runners were compared to 247 never-runner "couch potato" controls. The study showed that "older persons" who engage in vigorous running and other aerobic activities have lower mortality and slower development of disability (including osteoarthritis) than do members of the general population $[5,6]$. The association was probably related to increased aerobic activity, strength, fitness, and increased organ reserve rather than to an effect of postponed osteoarthritis development. 
We are less concerned about violinists, dancers, soccer players, and runners - what about the rest of us? Cell stress, damage, initiators of inflammation, and a host of age-related mechanisms (related to protein, carbohydrate, and lipid metabolism all abbreviated under the umbrella of "reactive oxygen species") have been implicated [7]. The appearance of cytokines such as tumor necrosis factor-alpha (TNF $\alpha)$, interleukin-1 (IL-1), transforming growth factor beta (TGF $\beta$ ), as well as disturbed sirtuin-1 function, will not surprise you.

Treatment options aside from platelet therapy and nonproven operative interventions are suboptimal and nonspecific. Nonsteroidal anti-inflammatory drugs (NSAIDS), cyclooxygenase- 1 and 2 inhibitors, acetaminophen, and aspirin are all employed. Not surprisingly, an anti-cytokine treatment similar as used in rheumatoid arthritis has also been advocated [8]. The entire "pain" industry is largely fed by osteoarthritis. Thus, the ramifications of this underappreciated condition are tremendous.

The pannexins are a family of vertebrate proteins with homology to the innexins. Innexins are responsible for forming gap junctions, while pannexins predominantly exist as large transmembrane channels that connect the intracellular and extracellular space [9]. Pannexin glycoproteins allow the passage of ions and small molecules including adenosine triphosphate (ATP) [10]. The pannexins have been implicated in purinergic receptor signaling and in the process of tumor development. Perhaps mouse models could provide more insight [11].

In this issue of $\mathrm{J} \mathrm{Mol} \mathrm{Med,} \mathrm{Moon} \mathrm{et} \mathrm{al.} \mathrm{have} \mathrm{done} \mathrm{exactly}$ that [12]. Pannexin-3 (Panx3) mediates ATP release in chondrocytes. The process accelerates hypertrophic differentiation. Chondrocyte hypertrophy is responsible for cartilage reabsorption in preparation for bone deposition. The master hypertrophic regulator RUNX2 targets Panx3. The transcription factor also drives matrix metalloproteinase-13 (MMP13) expression. MMP13 is a partner of Panx3. The authors generated both Panx3 gene-deleted (-/-) mice and cartilagespecific Panx3 gene-deleted mice. Panx $3-/-$ mice had no specific phenotype. The authors performed surgical destabilization of the medial meniscus (DMM). The DMM procedure that is analogous to a traumatic joint injury-induced osteoarthritis provides a reproducible model of osteoarthritis in mice [13]. Elimination of $P n x 3$ in cartilage led to a dramatic decrease in medial tibial plateau, medial-femoral condyle, and whole joint osteoarthritis with remarkable improvements in osteoarthritis-research-society-international (OARSI) joint injury scores. These findings potentially link pannexin channels to a human disease [14]. How could the process work?

The authors observed reduced MMP13 immunostaining after Panx 3 deletion [12]. Mechanistically, Runx 2 could mediate induction of Panx3 leading to increased ATP release from chondrocytes. Extracellular ATP could signal by means of the purine receptors, $\mathrm{P} 2 \mathrm{Y}$ a G protein-coupled receptor, and
P2X a gated ion channel. The resulting signals could lead to calcium signaling and ERK1/2 stimulated signaling thereby further activating Runx2. This state-of-affairs could begin a vicious cycle leading to further Runx 2 and MMP13 expression facilitating additional joint destruction. Pathway suggestions are clearly speculative (Fig. 1).

Information on pannexins in human tissues is rather sparse. Panx 1 and Panx 3 channels regulate skeletal muscle myoblast proliferation and differentiation including cells from humans [15]. The channels are expressed in human cartilage [16]. Panx3-transfected chondrocytic ATDC5 cells reduced parathyroid hormone-induced cell proliferation and promoted the release of ATP into the extracellular space, possibly by action of Panx 3 as a hemichannel. Panx 3 expression in chondrogenic ATDC5 cells reduced intracellular cAMP levels and the activation of cAMP-response elementbinding, a protein kinase A downstream effector. The Panx3 activities were blocked by anti-Panx 3 antibody. These results suggest that Panx 3 functions to switch the chondrocyte cell fate from proliferation to differentiation by regulating the intracellular ATP/cAMP levels [17]. The current studies will undoubtedly stimulate much human PANX3 research and possibly target purinergic receptors as potential treatment avenues for osteoarthritis. The clinical ramifications of this research area are enormous and the field should be watched with extreme interest!

Respectfully,

Friedrich C. Luft

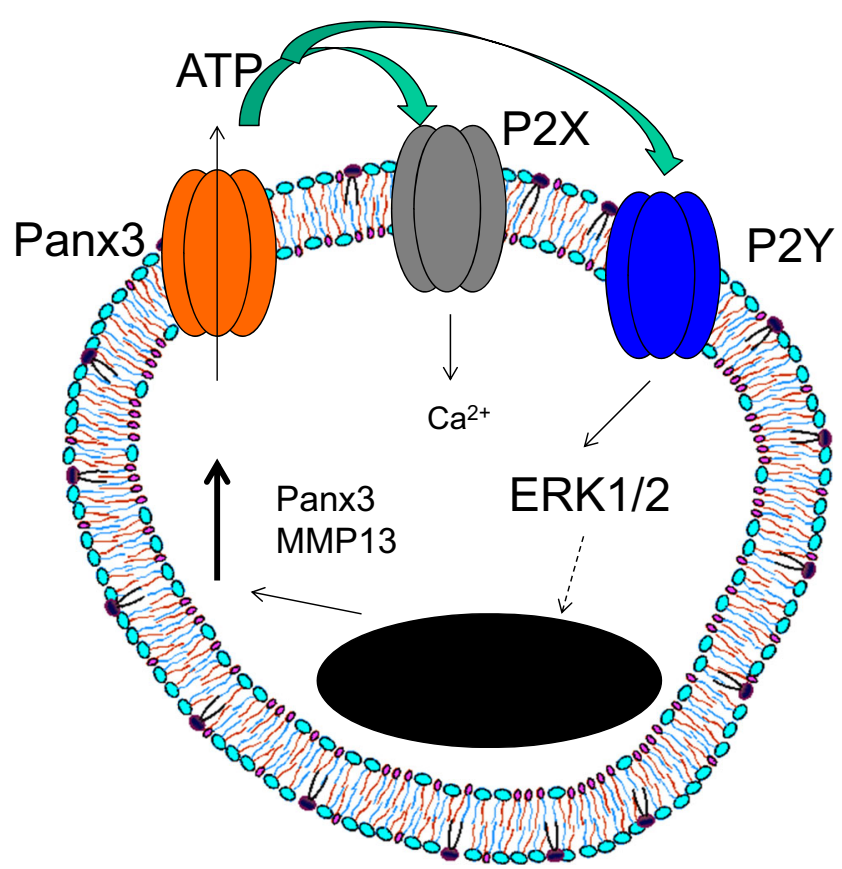

Fig 1 Entirely speculative notion on how the authors' findings might work. Panx3 is a glycoprotein ATP transporter, regulated by Runx2. ATP could signal over P2X and P2Y resulting in a positive feedback situation upregulating Panx3 and MMP13. MMP13 could contribute to osteoarthritis 


\section{References}

1. Lohmander LS, Englund PM, Dahl LL, Roos EM (2007) The longterm consequence of anterior cruciate ligament and meniscus injuries: Osteoarthritis. Am J Sports Med 35:1756-1769

2. Sanchez M, Anitua E, Orive G, Mujika I, Andia I (2009) Plateletrich therapies in the treatment of orthopaedic sport injuries. Sports Med 39:345-354

3. Staud R (2011) Evidence for shared pain mechanisms in osteoarthritis, low back pain, and fibromyalgia. Curr Rheumatol Rep 13:513-520

4. Rietveld AB (2013) Dancers' and musicians' injuries. Clin Rheumatol 32:425-434

5. Venes D (1995) Running and the development of disability with age. Ann Intern Med 122:475

6. Fries JF, Singh G, Morfeld D, Hubert HB, Lane NE, Brown BW Jr (1994) Running and the development of disability with age. Ann Intern Med 121:502-509

7. Loeser RF (2012) The effects of aging on the development of osteoarthritis. HSS J Musculoskelet J Hosp Spec Surg 8:18-19

8. Calich AL, Domiciano DS, Fuller R (2010) Osteoarthritis: can anti-cytokine therapy play a role in treatment? Clin Rheumatol 29:451-455

9. Litvin O, Tiunova A, Connell-Alberts Y, Panchin Y, Baranova A (2006) What is hidden in the pannexin treasure trove: The sneak peek and the guesswork. J Cell Mol Med 10:613-634
10. Good ME, Begandt D, DeLalio LJ, Keller AS, Billaud M, Isakson BE (2015) Emerging concepts regarding pannexin 1 in the vasculature. Biochem Soc Trans 43:495-501

11. Moon PM, Beier F (2015) Novel insights into osteoarthritis joint pathology from studies in mice. Curr Rheumatol Rep 17:524

12. Moon PM, Penuela S, Barr K, Khan S, Pin CL, Welch I, Attur M, Abramson SB, Laird DW, Beier F (2015) Deletion of Panx3 prevents the development of surgically induced osteoarthritis. J Mol Med. doi:10.1007/s00109-015-1311-1

13. Glasson SS, Blanchet TJ, Morris EA (2007) The surgical destabilization of the medial meniscus (dmm) model of osteoarthritis in the 129/svev mouse. Osteoarthr Cartil OARS Osteoarthr Res Soc 15:1061-1069

14. Penuela S, Harland L, Simek J, Laird DW (2014) Pannexin channels and their links to human disease. Biochem J 461:371-381

15. Langlois S, Xiang X, Young K, Cowan BJ, Penuela S, Cowan KN (2014) Pannexin 1 and pannexin 3 channels regulate skeletal muscle myoblast proliferation and differentiation. J Biol Chem 289: 30717-30731

16. Penuela S, Bhalla R, Gong XQ, Cowan KN, Celetti SJ, Cowan BJ, Bai D, Shao Q, Laird DW (2007) Pannexin 1 and pannexin 3 are glycoproteins that exhibit many distinct characteristics from the connexin family of gap junction proteins. J Cell Sci 120:3772-3783

17. Iwamoto T, Nakamura T, Doyle A, Ishikawa M, de Vega S, Fukumoto S, Yamada Y (2010) Pannexin 3 regulates intracellular atp/camp levels and promotes chondrocyte differentiation. J Biol Chem 285:18948-18958 